\title{
Efecto de la saturación en el deslizamiento de talud en la comunidad San Juan de Grijalva, Chiapas
}

\author{
The Effect of Saturation on the Slope Sliding \\ in the San Juan de Grijalva Comunity, Chiapas
}

\author{
Mora-Ortiz R.S. \\ Facultad de Ingeniería \\ Universidad Autónoma de Querétaro \\ Correo: renemora1121@hotmail.com
}

\author{
Rojas-González E. \\ Facultad de Ingeniería \\ Universidad Autónoma de Querétaro \\ Correo:erg@uaq.mx
}

Información del artículo: recibido: marzo de 2009, reevaluado: octubre de 2010, aceptado: febrero de 2011

\section{Resumen}

Algunos taludes que permanecen estables durante muchos años pueden presentar deslizamientos cuando ocurre un período de lluvia extraordinaria. En este fenómeno intervienen no sólo las características litológicas, geométricas y mecánicas del talud, sino también el régimen de lluvia-evaporacióninfiltración del sitio. En este artículo se analiza la estabilidad de un talud en la comunidad San Juan de Grijalva, municipio de Ostuacán, Chiapas, el cual presentó un deslizamiento durante un período de lluvias intensas. El volumen de este deslizamiento fue de más de 5 millones de metros cúbicos de suelo y ocasionó la obstrucción del cauce del río Grijalva. De este talud se obtuvieron sus características estratigráficas y geométricas y se realizó un muestreo inalterado para determinar las características mecánicas del material del talud. En el análisis de estabilidad se consideró la variación de la cohesión provocada por el humedecimiento y con ello se pudo observar cómo el factor de seguridad del talud evoluciona con el contenido de agua del cuerpo del talud. Mediante el análisis de la infiltración de agua de lluvia y de la estabilidad del talud ha sido posible reproducir el proceso de falla.

\section{Descriptores}

- talud

- estabilidad

- humedecimiento

- cohesión

- grado de saturación

- infiltración 


\begin{abstract}
A number of slopes that have been stable during many years may fail when an extraordinary rain period occurs. This phenomenon involves not only the lithology, the geometric and the mechanical characteristics of the slope but also the rain-evaporation-infiltration regime of the site. In this paper, the stability of a slope in the comunity of San Juan de Grijalva, Ostuacán, Chiapas (Mexico) that failed during an intense raining period is analyzed. The volume of this slide was over 5 millions of cubics meters of soil and it produced the obstruction of the Grijalva river. The stratigraphic and geometric properties of the slope were determined and undisturbed samples were obtained in the site to determine the mechanical properties of the material. The stability analysis considered the variation of the cohesion of the soil caused by wetting and it was possible to observe the evolution of the safety factor with the water content of the material. Through the analysis of the rain infiltration and the stability of the slope, it has been possible to reproduce the failure process.
\end{abstract}
Keywords
- slope
- stability
- wetting
- cohesion
- degree of saturation
- infiltration

\section{Introducción}

En México, durante cada época de lluvia ocurren deslizamientos de talud en algunas zonas del país. Estos deslizamientos causan pérdidas tanto económicas como en vidas humanas. Este fenómeno se ha visto incrementado debido al cambio climático que ha provocado la aparición de lluvias más intensas y de mayor duración.

En general, las fallas estructurales de taludes son el producto de una combinación de factores tales como el relieve, la geología, la historia tectónica, así como el intemperismo y la erosión a la que ha estado sometida la zona. Las fallas en taludes durante periodos prolongados de infiltración, son atribuidas al avance del humedecimiento dentro del cuerpo del talud. Como consecuencia de esto la resistencia al esfuerzo cortante producida por la succión se ve disminuida significativamente (Cho y Lee, 2001, 2002).

El objetivo de este artículo es proponer una metodología para determinar el efecto de la saturación en la estabilidad de taludes, utilizando los conocimientos sobre el flujo de agua, la resistencia y la estabilidad de taludes en suelos no saturados. Este procedimiento se aplicó al talud localizado en la comunidad de San Juan de Grijalva, Ostuacán, Chiapas (figura 1), el cual falló durante un periodo de lluvia intensa en el año 2007. Entre los factores principales de esta inestabilidad están las características litológicas, geométricas y mecánicas del talud, pero de manera preponderante están las condiciones climáticas y de drenaje de la región.

Por lo general, las fallas estructurales de talud que ocurren durante la época de lluvia tienen el mismo detonante. Para las fallas poco profundas, la superficie crítica de deslizamiento puede originarse por una de tres opciones: humedecimiento de la superficie del talud, incremento del nivel de agua subterránea o humedecimiento de la interfaz suelo-roca (Xie et al., 2004). Este tipo de fallas están relacionadas con periodos largos de lluvia con moderada intensidad y pueden generar presiones de poro positivas en el cuerpo del talud (Flores et al., 2003). Las fallas más profundas están relacionadas con la disminución de la succión (Collins y Znidarcic, 2004) y se deben a precipitaciones de corta duración pero de gran intensidad (Casagli et al., 2005). Es importante señalar que una gran parte de los deslizamientos de taludes tiene lugar en zonas tropicales, es decir, con precipitaciones frecuentes durante el año (Bujang, 2006); y pueden ocurrir en una gran variedad de suelos en climas tropicales y subtropicales (Tohari et al., 2007), presentándose tanto en taludes arenosos (Ching-Chuan et al., 2008) como en arcillosos (Cancelli, 1981).

\section{Localización y características del evento}

El municipio de Ostuacán se localiza en los límites de las montañas del norte y de la llanura costera del Golfo, predominando el relieve montañoso (figura 1). Sus coordenadas son $17^{\circ} 24^{\prime} \mathrm{N}$ y $93^{\circ} 20^{\prime} \mathrm{W}$.

El clima es cálido húmedo con lluvias todo el año, con una precipitación media anual de 1968.9 mm, situándose junto con Tabasco y el sur de Veracruz como las zonas con mayor precipitación en la República Mexicana.

Las rocas que componen los taludes de esta zona son generalmente muy permeables, es decir, permiten fácilmente el paso del agua, esta condición tiene sus ventajas y sus desventajas. La ventaja es que facilitan la infiltración de aguas meteóricas a los mantos freáticos y 


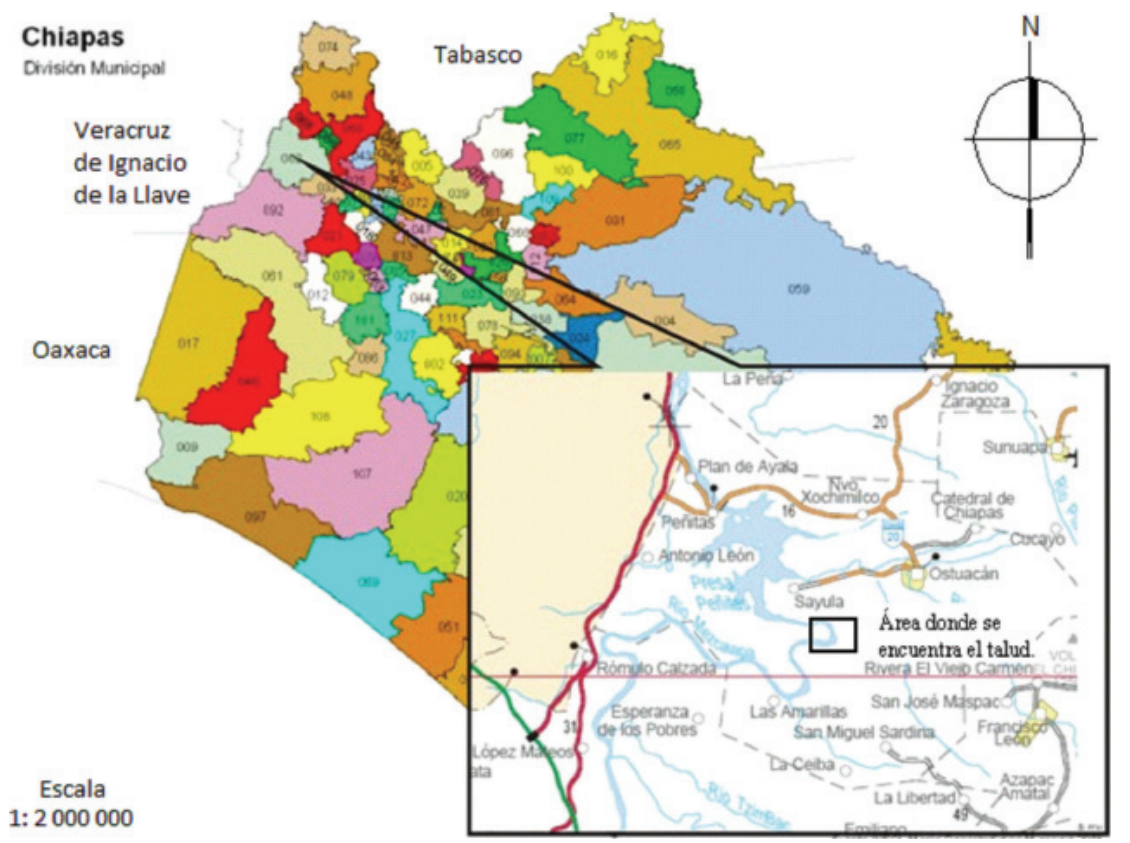

Figura 1. Localización del área de estudio. Fuente: INEGI zonas de recarga, pero la gran desventaja para este caso es que esa infiltración afecta al suelo constituyente de los taludes. Por otra parte, la cantidad de agua que corre sobre la superficie de los taludes provoca su erosión y la infiltración puede generar tubificación en suelos que no son autoestables y en algunos casos, puede generar presiones de agua positivas.

El domingo 4 de noviembre de 2007 a las 20:32 horas, en la comunidad San Juan de Grijalva, 2ª Sección de Ostuacán, ocurrió un deslizamiento de terreno que generó un alud de rocas, tierra y árboles, de aproximadamente 170 mil toneladas, el cual al precipitarse sobre el cauce del río Grijalva, provocó una ola de aproximada-

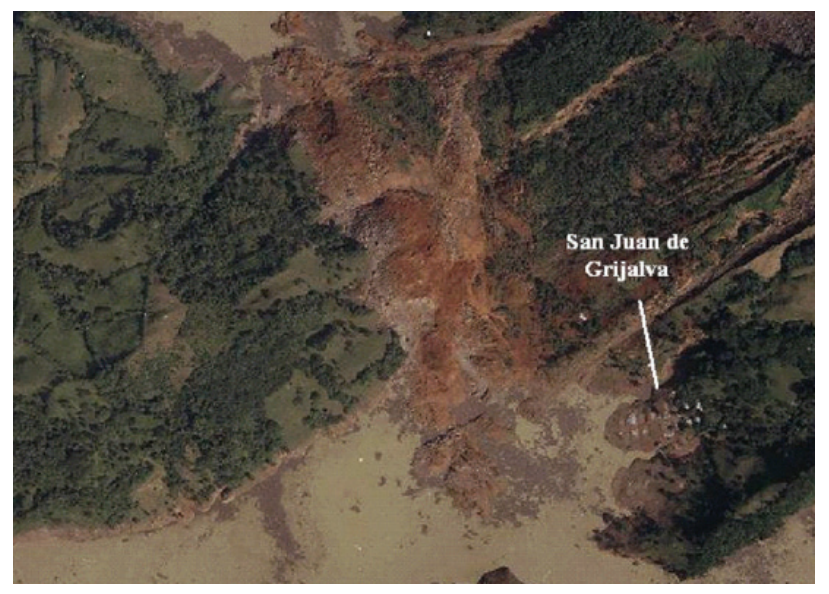

Figura 2. Fotografía aérea del deslizamiento de talud en la comunidad San Juan de Grijalva.

Fuente: Comisión Federal de Electricidad (CFE) mente 50 metros de altura y 5 kilómetros de extensión, inundando y destruyendo viviendas en la localidad y obstruyendo el cauce, como se observa en la figura 2 (Evaluación para Presidencia de la República, 2007).

\section{Metodología}

En este sitio se realizó la extracción de dos muestras inalteradas representativas del suelo que conforma el cuerpo del talud. De este material se obtuvieron las propiedades índice, peso especifico de sólidos, relación de vacíos, contenido de agua, grado de saturación, conductividad hidráulica, granulometría por vía seca y húmeda, esfuerzo de preconsolidación y parámetros de resistencias (ángulo de fricción $(\phi)$, cohesión (c)) mediante pruebas triaxiales realizadas en el laboratorio de Mecánica de Suelos de la Universidad Autónoma de Querétaro.

También, se obtuvo la geometría del talud antes del deslizamiento, con información proporcionada por la Comisión Federal de Electricidad (CFE). Una vez que se obtuvieron las propiedades mecánicas e hidráulicas del material, se realizó una modelación del comportamiento mecánico del talud antes y durante el periodo de lluvias utilizando dos programas de cómputo especializados. El primero para simular el flujo de agua en suelos no saturados (PlaxFlow, 2003a) y el segundo en el análisis de la estabilidad de taludes (Geo, 1995). Las características del material empleado aparecen en la tabla1. La granulometría del material se muestra en la figura 3. 
Tabla 1. Propiedades del suelo

\begin{tabular}{cccccccc}
\hline L.L. (\%) & 51 & L.P. (\%) & 36 & I.P.(\%) & 16 & SUCCS & MH \\
\hline$\gamma_{\mathrm{m}}$ & 16.37 & $\mathrm{Ss}$ & 2.70 & $\mathrm{e}$ & 1.32 & $\mathrm{~W}(\%)$ & 41.9 \\
$\mathrm{GW}(\%)$ & 85.54 & $\mathrm{Ks}$ & $8.95 \mathrm{E}-05$ & $\mathrm{Pc}$ & 435.49 & & \\
\hline
\end{tabular}

Simbologia:

L.L. límite líquido,

L.P. límite plástico,

I.P. índice de plasticidad,

SUCCS Sistema Unificado de Clasificación de Suelos, (MH) limo de alta compresibilidad,

Ss Peso específico relativo de sólidos,

$\gamma_{\mathrm{m}} \quad$ Peso específico de la masa de suelo $\left(\mathrm{kN} / \mathrm{m}^{3}\right)$,

e Relación de vacíos,

$\mathrm{W}(\%)$ humedad natural,

Gw $(\%)$ Grado de saturación natural,

Ks conductividad hidráulica $(\mathrm{cm} / \mathrm{seg})$,

Pc carga de preconsolidación $\left(\mathrm{kN} / \mathrm{m}^{2}\right)$.

La conductividad hidráulica en un suelo no saturado no es una constante, sino que es una función de la succión. Por lo tanto, primero es necesario determinar la relación entre succión y grado de saturación. A esta relación se le denomina curva de retención (Jaehong et al., 2004). A su vez, de esta curva se puede obtener la conductividad hidráulica del material en función de la succión, ya que la curva de retención puede proporcionar una estimación de la distribución de los tamaños de poros del suelo (Pires et al., 2008). La curva de retención se obtuvo por el método del papel filtro. Para ello, primeramente se realizó la calibración del papel y los resultados se muestran en la figura 4 . El papel utilizado fue marca Schleicher y Shuell, numero 589 y se calibró de acuerdo al siguiente procedimiento: se prepararon soluciones de agua destilada con diferentes concentraciones de sal, lo cual proporciona una succión conocida. Dichas soluciones se colocaron dentro del contenedor de vidrio. Además se colocaron dos papeles filtro inmediatamente por encima de la solución sobre una canastilla de alambre y se selló el contenedor herméticamente. Después se colocó el contenedor en una caja aislada térmicamente y se introdujo en un cuarto de temperatura controlada en donde se permitió el equilibrio de la humedad relativa entre el papel y la solución por espacio de 7 días. Una vez terminado el periodo de equilibrio, se determinó la humedad de los papeles filtro y se graficaron los datos obtenidos según el procedimiento des-
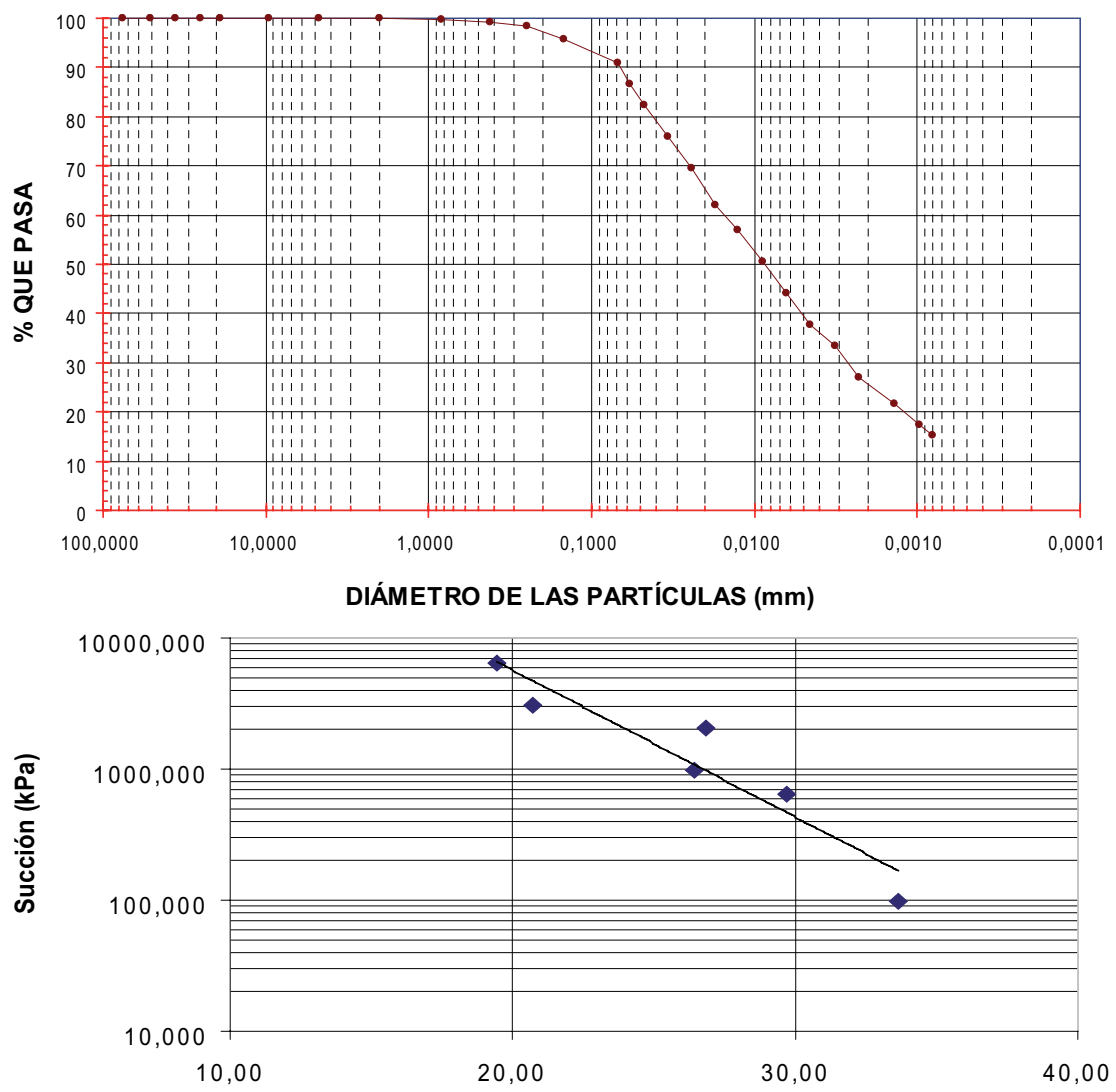

Contenido de Humedad (\%)
Figura 3. Granulometría vía humedad

Figura 4. Curva de calibración (succión vs contenido de humedad) 
crito por la norma ASTM D 5298-94 (1994). Con esta calibración es posible determinar la succión de muestras de suelo con distintos grados de saturación para lo cual basta con realizar sobre la muestras el mismo procedimiento de papel filtro y utilizar la gráfica de calibración. Con este procedimiento se obtuvo la curva de retención en humedecimiento (figura 5).

Se realizaron ensayes triaxiales consolidados-drenados, con suelo saturado, para los cuales, se utilizaron 3 especímenes de suelo de $3.5 \mathrm{~cm}$ de diámetro y $9 \mathrm{~cm}$ de alto, se colocó cada una de las muestras en la base de una cámara triaxial, se cubrieron con una membrana de látex, ligada perfectamente al cabezal y la base de la cámara para evitar que penetre el agua que se utilizó para dar confinamiento al suelo. A cada muestra se le aplicaron esfuerzos confinantes de $49.03 \mathrm{kN} / \mathrm{m}^{2}, 98.07 \mathrm{kN} /$ $\mathrm{m}^{2}$ y $147.10 \mathrm{kN} / \mathrm{m}^{2}$. Se utilizó una carga axial en incrementos, permitiendo el drenaje a la muestra en todo momento, la velocidad de aplicación fue de $0.01 \mathrm{~mm} /$ min, tomándose lecturas de unidades de carga y unidades de deformación, hasta que se registraron dos unidades de carga iguales (Juárez y Rico, 1998).
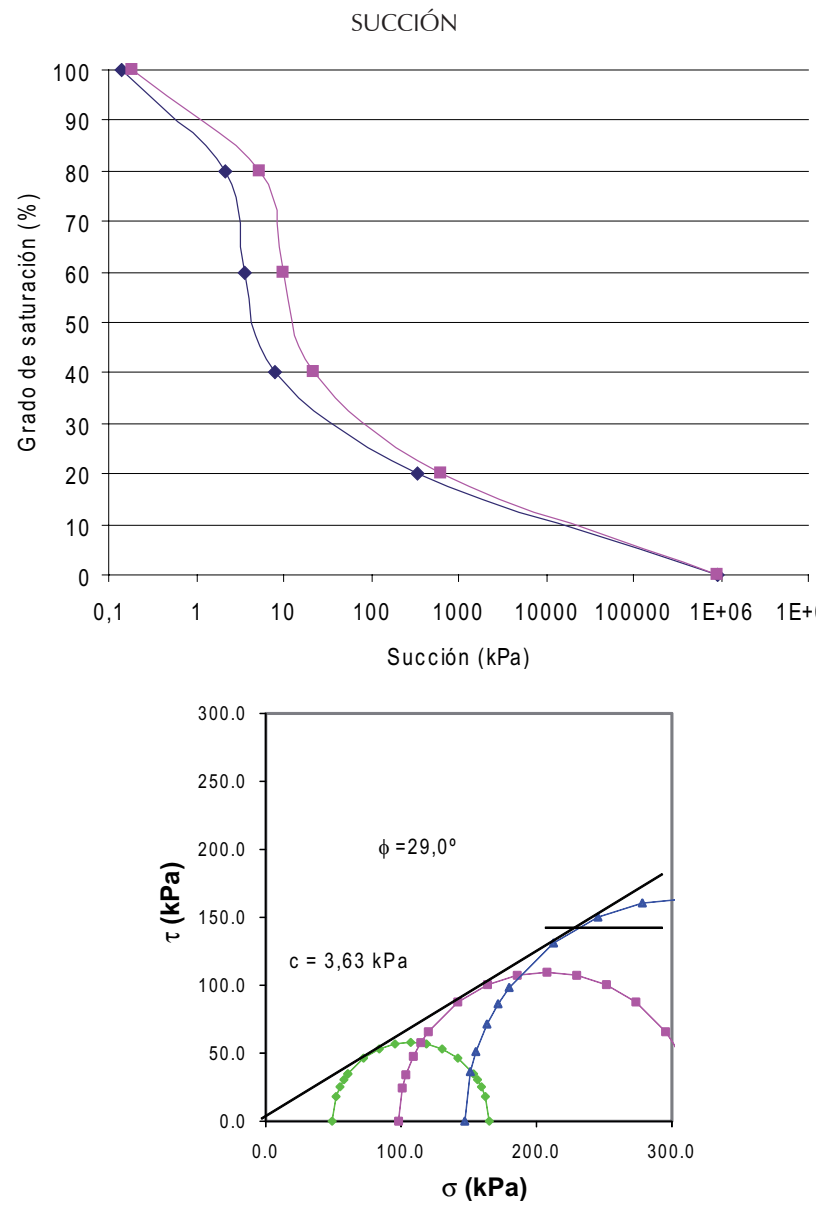

Posteriormente, con las lecturas tomadas se elaboraron los círculos de Mohr para la determinación del ángulo de fricción interna del suelo $(\phi)$, los cuales se muestran en la figura 6 en donde: $c$ representa la cohesión, $\phi$ es el ángulo de fricción interna del material, $\tau$ es el esfuerzo cortante y $\sigma$ el esfuerzo normal

De la misma manera, se realizaron ensayes triaxiales consolidados-drenados a diferentes grados de saturación y con un esfuerzo confinante de $147.10 \mathrm{kN} / \mathrm{m}^{2}$. Los diferentes grados de saturación de las probetas ensayadas se obtuvieron del humedecimiento de muestras totalmente secas (figura 7). Con los valores obtenidos del desviador a la falla, se construyeron los respectivos círculos de Mohr (figura 8).

Se obtuvieron datos de precipitación pluvial de la zona (CONAGUA), con la finalidad de realizar la modelación del flujo de agua en el cuerpo del talud, dichos datos se muestran el la figura 9, donde también se observan los días de mayor precipitación registrados en la estación Ocotepec: 28 de octubre con 403.4 $\mathrm{mm}, 29$ de octubre con $308.9 \mathrm{~mm}$ y 30 de octubre con $250.5 \mathrm{~mm}$.
Figura 5. Curva de retención en humedecimiento y secado (succión vs grado de saturación)

Figura 6. Resultados de los ensayes triaxiales saturados 


\section{Ensayes triaxiales (Humedecimiento)}

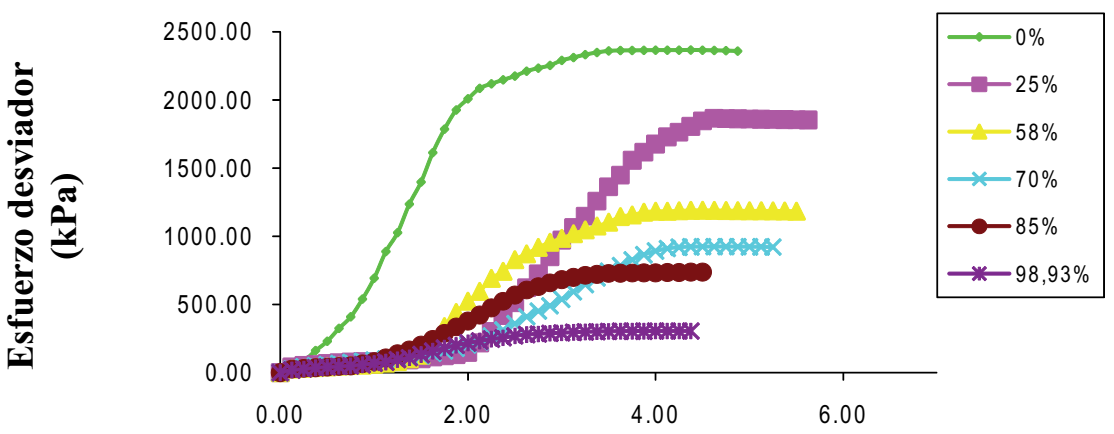

Deformación Unitaria (\%)
Figura 7. Esfuerzo desviador vs deformación unitaria, ensayes triaxiales con diferentes grados de saturación en humedecimiento

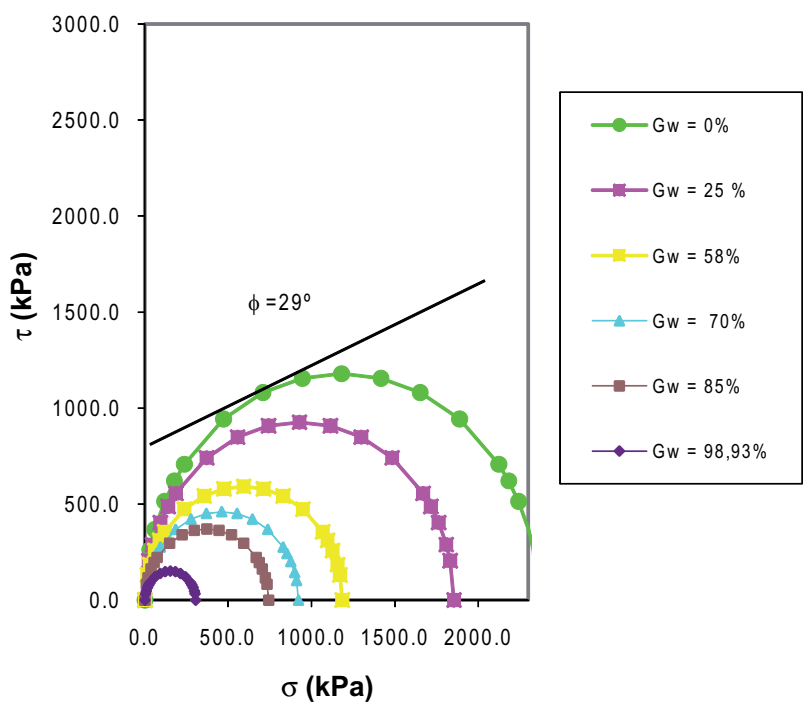

Figura 8. Círculos de Mohr de ensayes triaxiales a diferentes grados de saturación en humedecimiento, con un mismo confinante $\left(147.10 \mathrm{kN} / \mathrm{m}^{2}\right)$

\section{Resultados}

\section{Análisis de estabilidad}

Para el análisis de la estabilidad estructural del talud se utilizó el software Geo-Slope (1995), alimentado por los siguientes parámetros de entrada: peso específico del material $\gamma_{\mathrm{m}}$ en $\mathrm{kN} / \mathrm{m}^{3}$, cohesión c en $\mathrm{kPa}$ y ángulo de fricción interna $\phi$ en grados.

El Geo-Slope (1995) es un software de uso internacional con el que se puede analizar la estabilidad de taludes de suelo e incluso de roca. Permite entre otras cosas incluir en el cálculo varias capas de diversos materiales, la presencia del nivel freático y diversas condiciones de carga, además de que toma en cuenta la presión de poro. Todo lo anterior hace de este programa uno de los mejores para este análisis.
Nota: los días que no se muestran en la figura no tuvieron precipitación. Fuente: CONAGUA

Precipitación año 2007

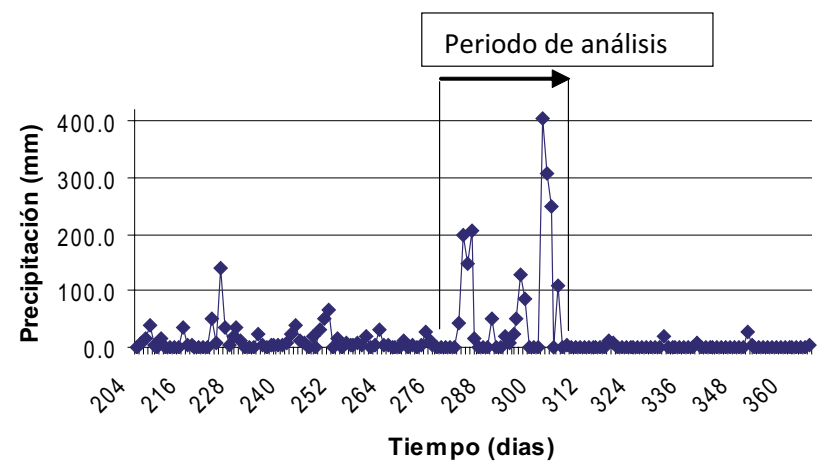

Figura 9. Precipitación pluvial registrada en la estación Ocotepec

El factor de seguridad es calculado con el método de las dovelas de Fellenius, por lo cual se muestra la zona que se desliza dividida en dovelas y en otro color. El resultado de cada análisis muestra una malla donde cada punto es el radio de un posible círculo de falla, mostrando el valor del círculo con el menor factor de seguridad.

El análisis se realizó con los datos del perfil del talud en la zona media del deslizamiento.

Tomando como constante el ángulo de fricción interna del suelo $(\phi)$ obtenido de los ensayes triaxiales saturados (figura 6) y utilizando los círculos de Mohr para diferentes grados de saturación (figura 8), se determinó el valor de la cohesión para diversos grados de saturación (Fredlund y Rahardjo, 1985). Esta relación se puede observar en la figura 10. 


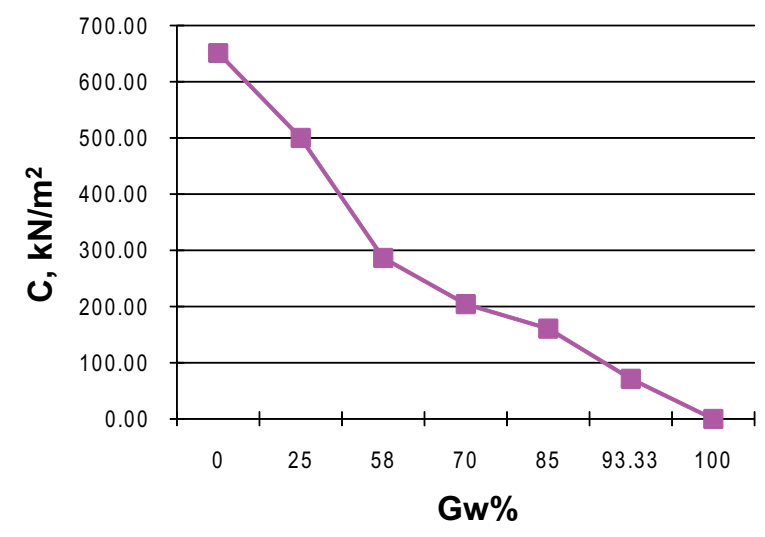

Se consideró una falla de tipo traslación. En este caso, se realizaron una serie de análisis haciendo variar el valor del grado de saturación del material. La variación se hizo desde el estado seco hasta el estado saturado. Con la ayuda de la curva característica en la trayectoria de humedecimiento (figura 5) se pudo determinar la succión existente en el talud para cada grado de saturación (figura 11). De acuerdo con este
Figura 10. Cohesión (c) vs Grado de saturación (Gw)

análisis, se puede clasificar al talud como de alto riesgo $(\mathrm{Fs}<1.1)$, de bajo a mediano riesgo $(1.1<\mathrm{Fs}<1.5)$ o sin riesgo ( $\mathrm{Fs}>1.5)$.

En función del factor de seguridad se realizaron las gráficas que relacionan el factor de seguridad con el grado de saturación (figura 12) y el factor de seguridad con la succión (figura 13).
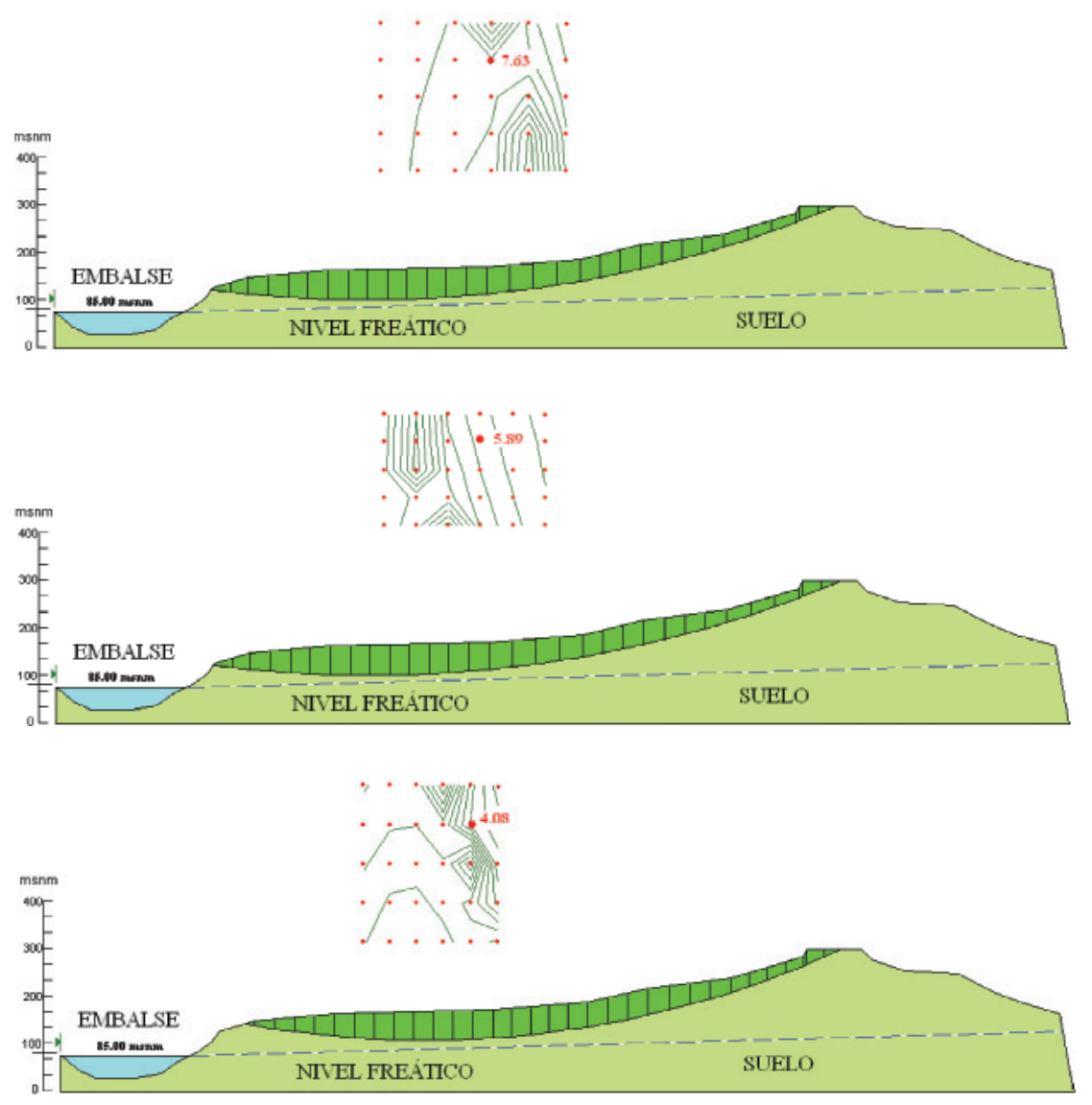

a)

$\mathrm{Gw}=0.00(\%)$,

$\mathrm{FS}=7.63$,

$\mathrm{s}=894619.255 \mathrm{kPa}$

(b)

$\mathrm{Gw}=25(\%)$,

$\mathrm{Fs}=5.89$,

$\mathrm{s}=263.024 \mathrm{kPa}$

Figura 11. Análisis de la estabilidad del talud a diferentes grados de saturación $\mathrm{Gw}(\%)=$ Grado de saturación, en porcentaje, Fs $=$ Factor de seguridad, s = Succión, en rama de humedecimiento (continúa) 

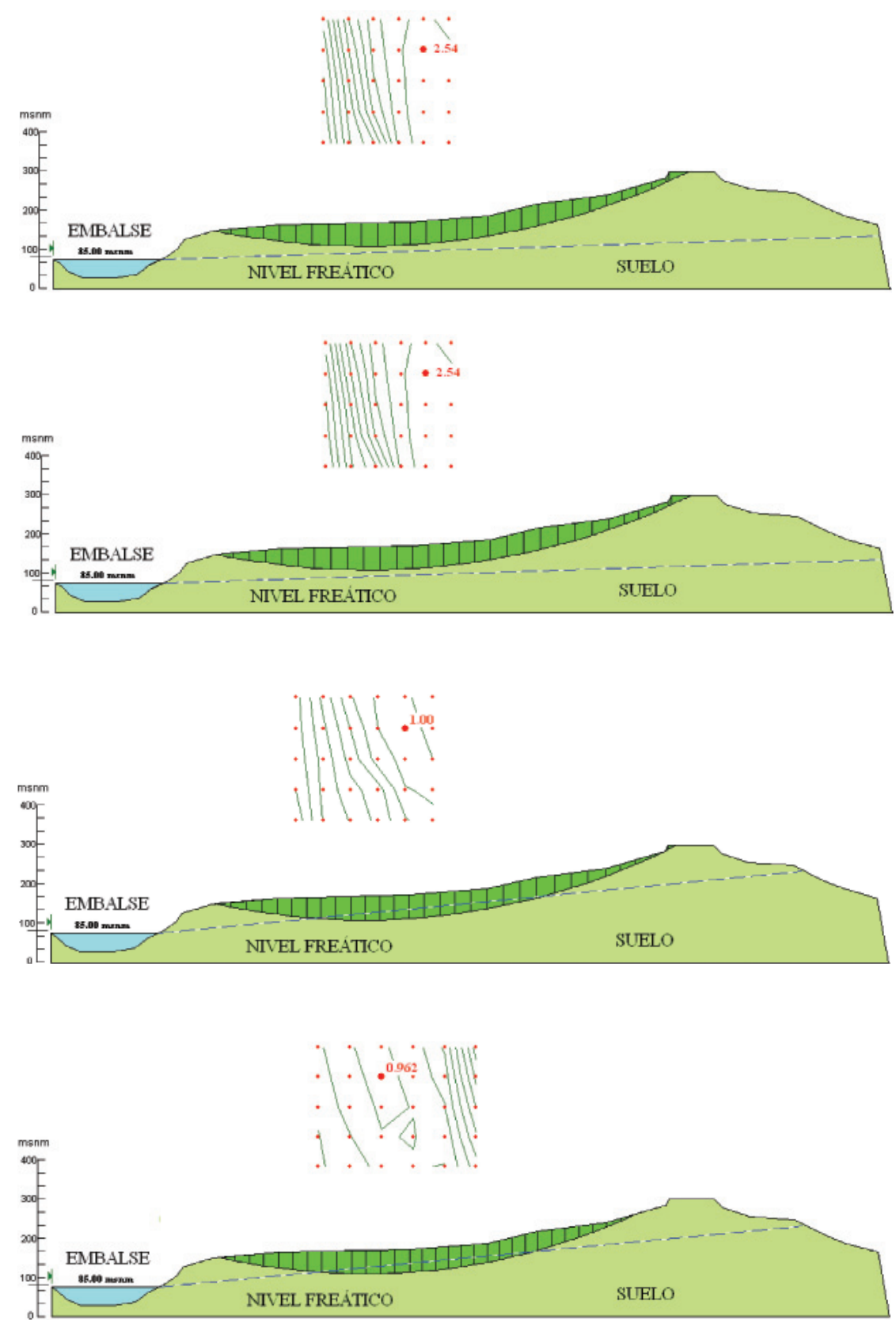

(d)

$\mathrm{Gw}=70(\%)$,

$\mathrm{Fs}=2.54$,

$\mathrm{s}=2.91 \mathrm{kPa}$ (e) $\mathrm{Gw}=85(\%)$, $\mathrm{Fs}=1.68$, $\mathrm{s}=1.691 \mathrm{kPa}$

$(\mathrm{f})$ $\mathrm{Gw}=98.41(\%)$ $\mathrm{Fs}=1.00$, $\mathrm{s}=0.305 \mathrm{kPa}$

Nota: factor de seguridad $=1$, es considerado como falla estructural. La línea punteada representa el NAF

Figura 11 (Continuación). Análisis de la estabilidad del talud a diferentes grados de saturación Gw(\%) = Grado de saturación, en porcentaje, Fs = Factor de seguridad, s = Succión, en rama de humedecimiento 

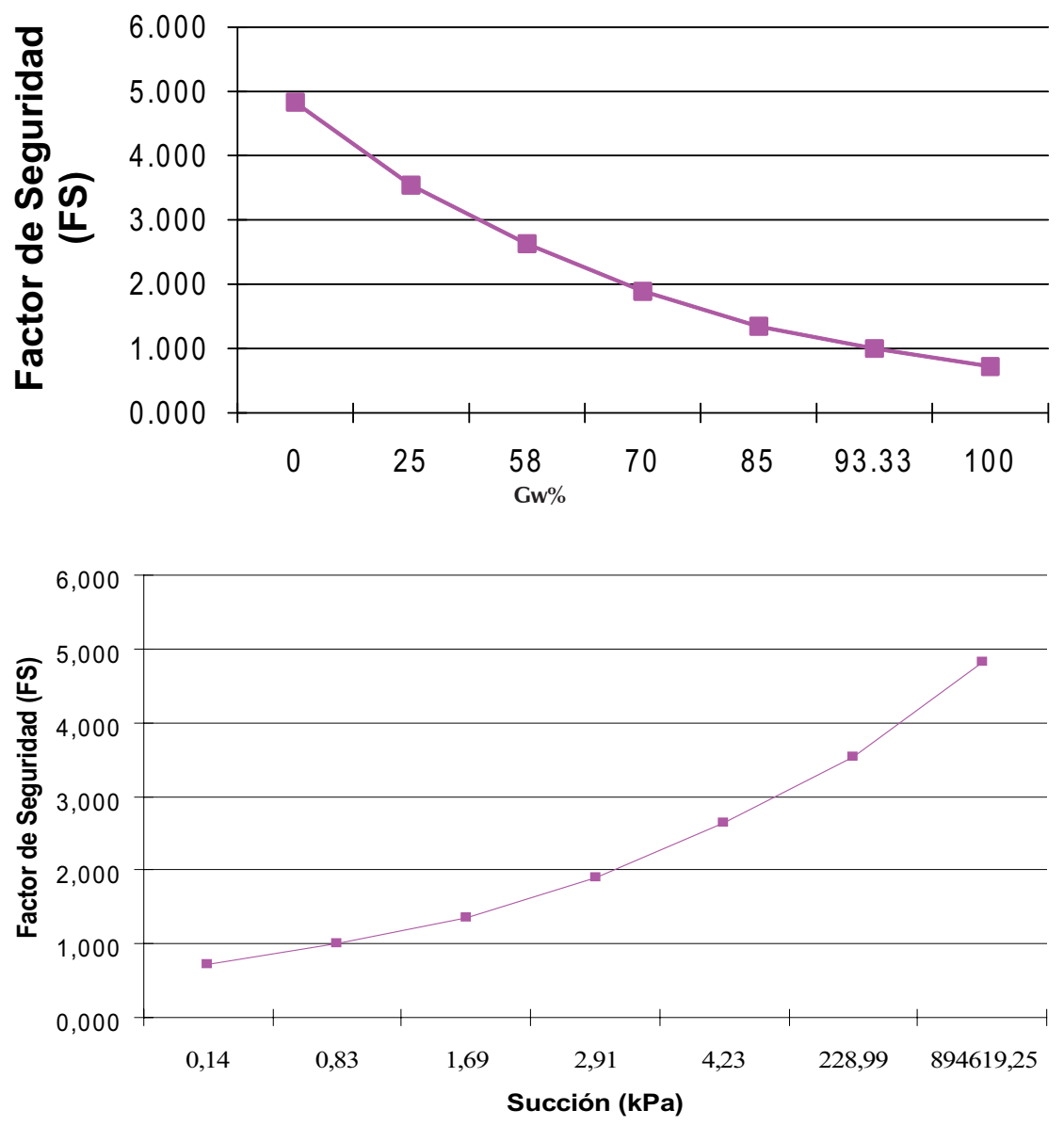

Figura 12. Factor de seguridad vs Grado de saturación $\left(\mathrm{G}_{\mathrm{w}}\right)$

Figura 13. Factor de seguridad vs Succión

\section{Flujo de agua en el talud}

Las fallas estructurales del talud, no sólo dependen de las características de resistencia del suelo, sino también de sus propiedades hidráulicas, de ahí la importancia del flujo de agua a través del talud (Collins y Znidarcic, 2004). La infiltración del agua producto de la precipitación pluvial básicamente puede ser de dos tipos: inundación de la superficie del suelo y la infiltración controlada (Alonso et al., 1995). La infiltración que se presentó es de tipo controlada y en este caso, la inclinación del talud es un factor determinante (Jaehong et al., 2004).

Mediante el programa de cómputo PlaxFlow (2003a), se simuló el flujo de agua en el cuerpo del talud, con lo cual, se pudieron observar las zonas en donde se infiltra el agua, así como la profundidad de la infiltración (figura 14).

Para la simulación del flujo de agua en el cuerpo del talud, además de los parámetros físicos y mecánicos, se determinaron las propiedades hidráulicas básicas de suelos, con los que se alimentó el software: Coeficiente de permeabilidad saturado (ks); Grado de Saturación $(\mathrm{Gw})$; Succión $(\psi)$.

PlaxFlow (2003a) es un programa de cómputo que utiliza el método de elementos finitos para modelar el flujo de agua establecido o transitorio en suelos no saturados utilizando la ecuación de Van Genuchten para calcular la conductividad a partir de la curva de retención, la cual juega un rol esencial en la determinación de las características hidráulicas del suelo. Además el software toma en cuenta la relación entre la presión de poro, el grado de saturación, el tamaño de las partículas y el coeficiente de permeabilidad, lo que hace que dentro de los programas comerciales PlaxFlow sea uno de los más completos para este análisis.

Entre los modelos matemáticos desarrollados, el modelo de Van Genuchten históricamente ha sido el más ampliamente adoptado. Sus tres parámetros $(\alpha, \beta$ y $\delta)$ son la clave para la aplicación de este modelo:

$k r=\frac{\left[1-(\alpha \psi)^{\beta-1}\left(1+(\alpha \psi)^{\beta}\right)^{-\delta}\right]^{2}}{\left(1+(\alpha \psi)^{\beta}\right)^{\delta / 2}}$ 
Donde:

$$
\begin{aligned}
\mathrm{kr}= & \begin{array}{l}
\text { conductividad hidráulica relativa de Van Ge- } \\
\text { nuchten, }
\end{array} \\
\psi= & \text { succión, } \\
\alpha, \beta, \delta= & \text { los parámetros, pero se considera que } \\
& \delta=(\beta-1) / \beta .
\end{aligned}
$$

Van Genuchten y otros (PlaxFlow, 2003b) propusieron un método de optimización por el método de mínimos cuadrados para determinar los parámetros anteriores (Cervantes, 2001).

Se utilizó una evaporación constante para zona tropical de $0.002 \mathrm{~m} /$ día. PlaxFlow utiliza una red de elementos finitos para el análisis de la infiltración, la cual es considerada solo en una dirección. La tasa de infiltración es calculada a través de los registros de precipita- ción diaria en mm en la zona, de la curva de retención de agua, de la permeabilidad, de la pendiente del terreno y la vegetación de la zona.

Además utiliza los siguientes parámetros: tensor de permeabilidad saturada $\left(\mathrm{K}_{\mathrm{sat}}\right)$, grado de saturación máxima y residual $\mathrm{S}_{\text {sat }} \mathrm{y}_{\text {residu' }}$ respectivamente. Los valores de dichos parámetros para este caso fueron los propuestos por el software para una arcilla: $0.077 \mathrm{~m} /$ día, 1 y 0.23 , respectivamente. El grado de humedad que se usó para iniciar la simulación fue el que tenía el suelo al momento de hacerse los muestreos y se consideró homogéneo para toda la masa de suelo localizada por encima del nivel freático.

En la figura 14 podemos observar el proceso de saturación en el cuerpo del talud, la barra localizada en la parte derecha de cada figura muestra el rango del grado de saturación.
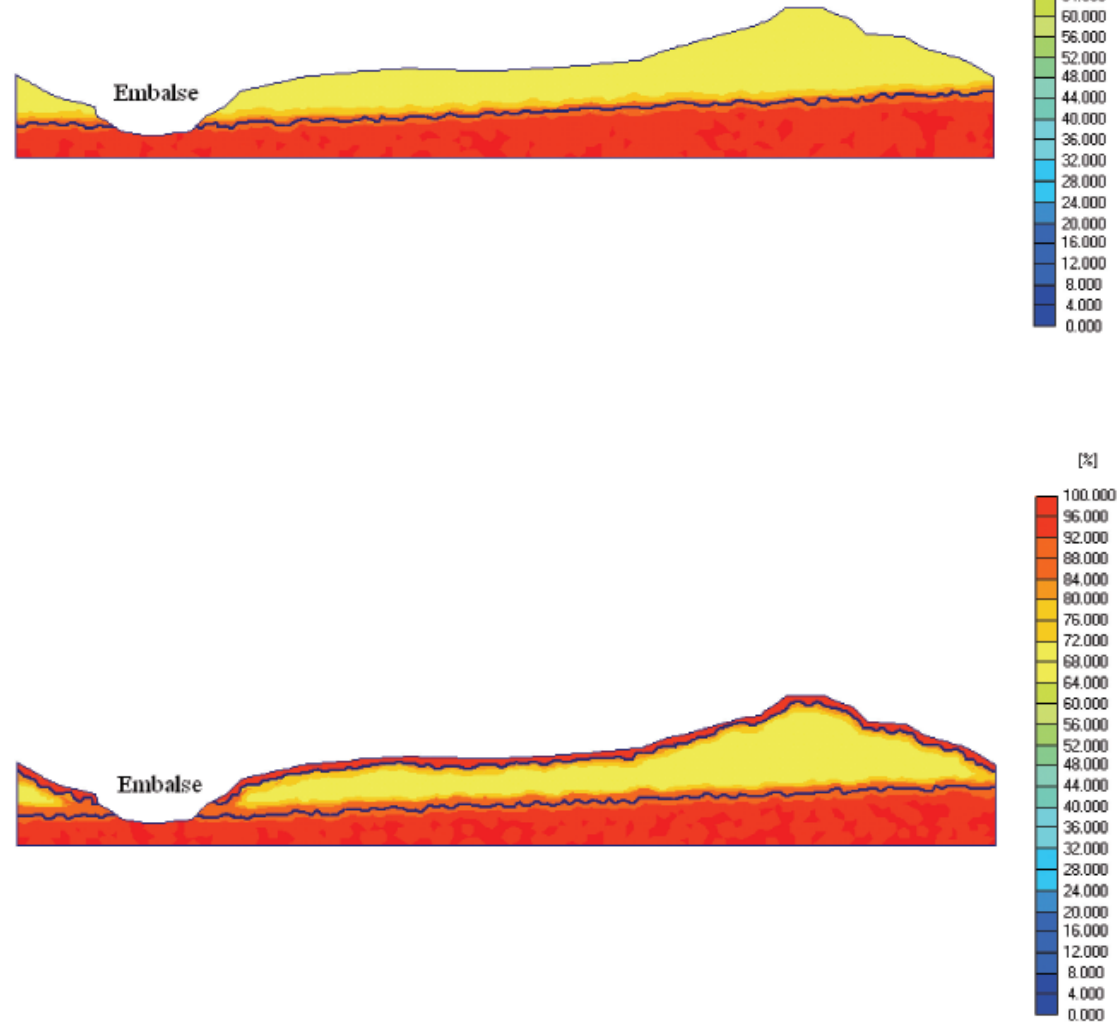

a) Estado del talud antes de temporada de lluvias

b) 5 de octubre 2007

Figura 14. Simulación de flujo de agua en el talud (continúa) 


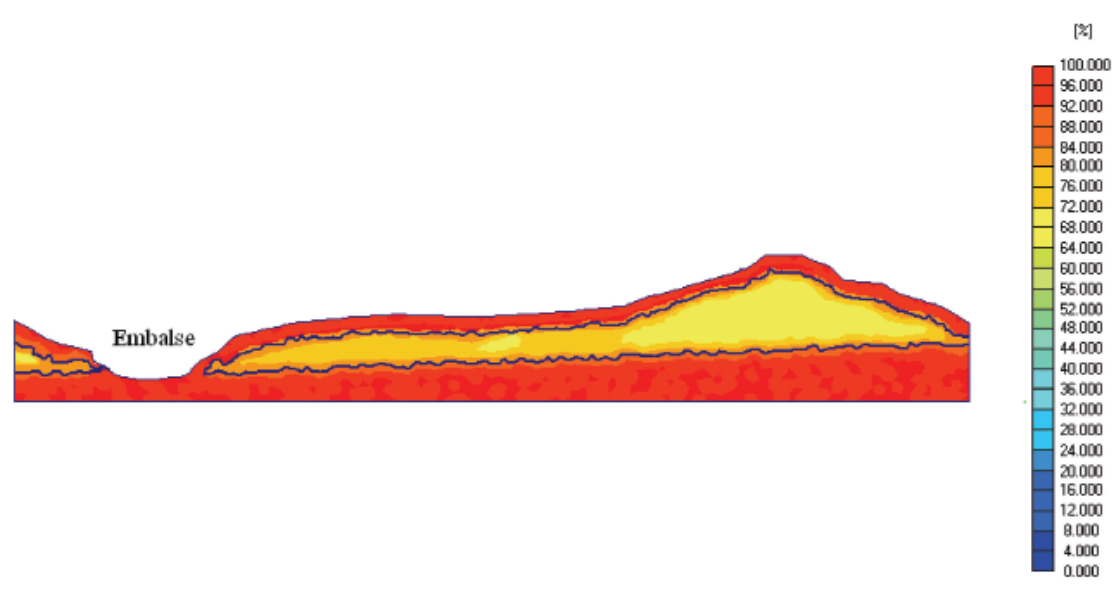

c) 15 de octubre 2007

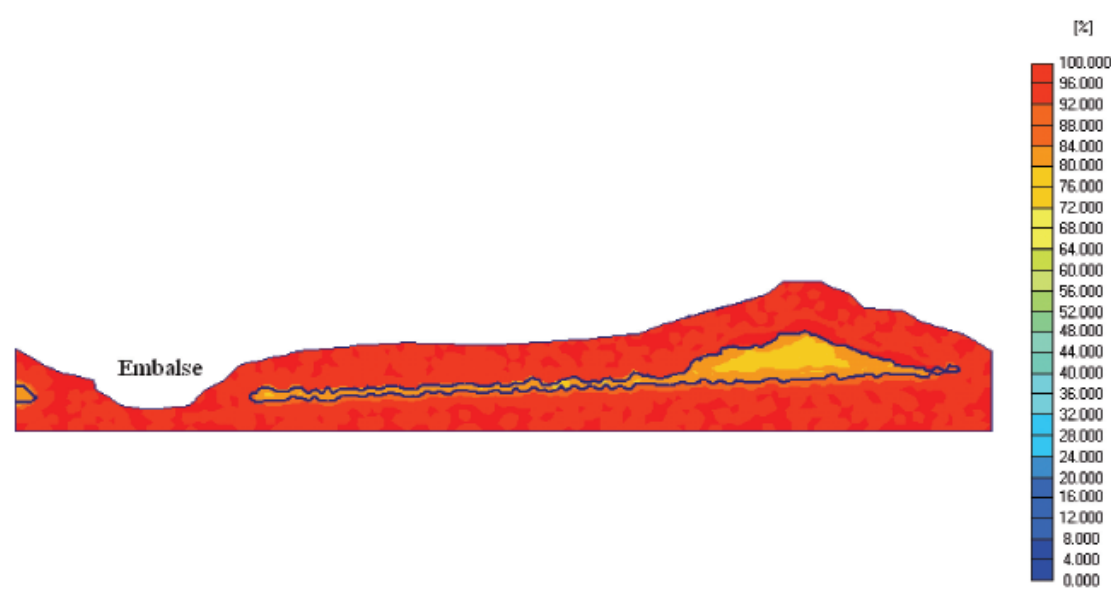

d) 25 de octubre de 2007

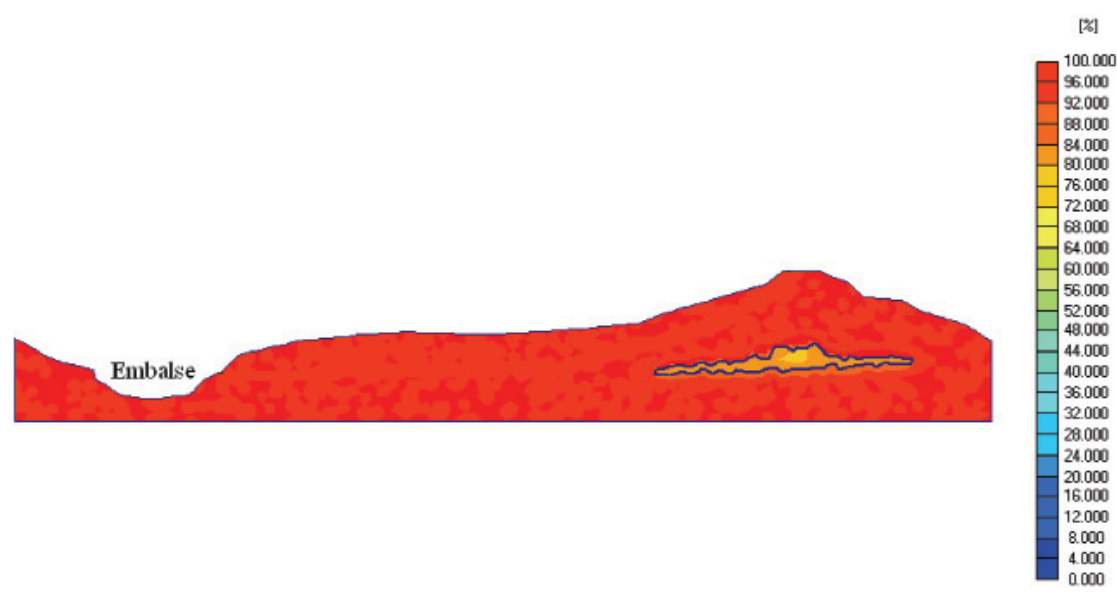

e) 4 de noviembre de 2007

Figura 14 (Continuación). Simulación de flujo de agua en el talud 
En la figura 15 se observa que al momento del deslizamiento, la superficie de falla real reportada en campo (CFE, 2008) se encuentra completamente inmersa dentro de la zona saturada, por lo que el factor de seguridad alcanza el valor de 1 de acuerdo al análisis de estabilidad (figura 11-f).

Los resultados de la simulación de flujo de agua presentados en la figura 14 se pueden comparar con los obtenidos para el análisis de estabilidad con un factor de seguridad de uno. El estado inicial del suelo corresponde al de la humedad natural en la que se tomaron las muestras. A partir de este momento, esta comparación muestra que para un periodo de 34 días de lluvia (1 de octubre - 4 de noviembre) la infiltración de agua prácticamente coincide con la superficie de falla, de tal manera que la falla del talud ocurriría a los 4 primeros días del mes de noviembre, lo cual coincide con los reportes sobre la falla del talud. Días antes del 4 de noviembre se observó una zona de inestabilidad que crecería conforme la infiltración de agua se incrementaba.

Para prevenir este fenómeno en taludes naturales es necesario encauzar correctamente el agua de lluvia por medio de drenes en la parte superior del talud y contracunetas que conduzcan el agua hacia la parte baja, minimizando la infiltración al cuerpo del talud. En casos críticos resulta necesario combinar esta solución con el uso de una membrana impermeable que puede ser plástica o a base de una malla metálica y concreto lanzado. En estos casos es necesario implementar un sistema de drenes en la cara protegida. Algunos autores recomiendan reforzar al talud por medio de la colocación de vegetación (árboles) de raíces profundas, debido a que dichas raíces pueden atravesar la zona potencial de falla y así contribuir a su estabilización (Danjon et al., 2008). En combinación, se pueden usar plantas como la Leucaena leucocephala para prevenir la erosión del talud debido al flujo superficial del agua (Normaniza et al., 2008).

Este trabajo muestra la metodología que puede emplearse para determinar el riesgo de deslizamiento de un talud ante un evento de lluvias extraordinarias. Tal metodología consiste en:

a) Identificar las zonas sensibles de deslizamiento en las comunidades o tramos carreteros. Se recurrirá a planos topográfico aéreos de la zona para ubicar los sitios que se consideren con mayor peligro.

b) Una vez identificadas dichas zonas, se deberá realizar un recorrido de los sitios para recabar la siguiente información: geometría del talud, construcciones aledañas, condiciones de drenaje, vegetación, clima, estratigrafía, nivel freático, impacto de la falla en el aspecto económico y de vidas humanas.

c) Se obtendrán muestras alteradas e inalteradas representativas de los materiales que conforman el talud para realizar los ensayes de laboratorio necesarios.

d) En el laboratorio se obtendrá el ángulo de fricción interna de cada material en condiciones saturadas mediante ensayes de corte directo aplicando tres valores de carga normal representativas del sitio. También pueden realizarse ensayes triaxiales consolidados-drenados o consolidados-no drenados con medición de la presión de poro, aunque estos últimos ensayes resultan más complicados por el tamaño de las muestras que se deben labrar y el procedimiento del ensaye en sí.

e) Se realizará el análisis de la estabilidad del talud en condiciones críticas, es decir, cuando el material se encuentra saturado.

f) Se obtendrán los registros de lluvia de la región. El análisis de la intensidad de lluvia que puede causar la falla del talud se puede realizar con un programa de flujo de agua en medios no saturados. En tal caso también se requerirá la realización de ensayes de corte directo con muestras sometidas a diversos grados de saturación.

Por medio de este procedimiento se pudo evaluar la estabilidad del talud antes y después del periodo de lluvias, y mediante el análisis de flujo en suelos no saturados, se verificó que la zona que se deslizó, efectivamente presentaba altos grados de saturación. Este procedimiento permite no sólo analizar deslizamientos ocurridos y verificar la influencia del humedecimiento en ellos, sino también analizar taludes con alto riesgo de deslizamiento. Esto permitiría prevenir este fenómeno al menos en las zonas más sensibles como son las zonas urbanas o rurales densamente pobladas y autopistas o caminos con gran afluencia de vehículos, y en

Superficie de deslizamiento real

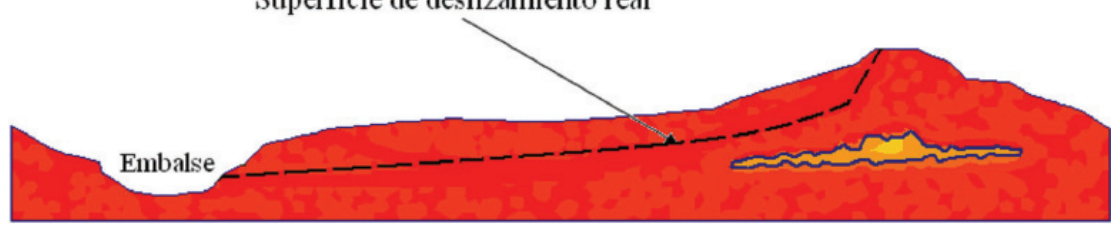

Figura 15. Localización de la superficie real de falla en el flujo de agua en el cuerpo del talud. Donde se observa que la zona de falla está localizada en la zona saturada (zona roja) 
el mejor de los casos, evitar la pérdida de vidas humanas. Así mediante el monitoreo de los taludes en la épocas de lluvia y con la aplicación del procedimiento descrito en esta investigación se podrán tomar las medidas necesarias para emitir oportunamente avisos de peligro a la población.

\section{Conclusiones}

Mediante algunas pruebas de laboratorio y el análisis de la estabilidad estructural del talud, se ha podido establecer la relación entre el grado de saturación del suelo y el factor de seguridad del talud analizado.

Este análisis demuestra que el humedecimiento del suelo fue la causa del deslizamiento de tierra en la comunidad de San Juan de Grijalva, Chiapas. Así las precipitaciones registradas entre los días 28 de octubre y 4 de noviembre de 2007, fueron el detonante del deslizamiento. El resultado de la modelación coincide aproximadamente con la fecha en que ocurrió el evento, así como con el volumen del desprendimiento (55 millones de metros cúbicos aproximadamente) y su longitud $(320 \mathrm{~m})$.

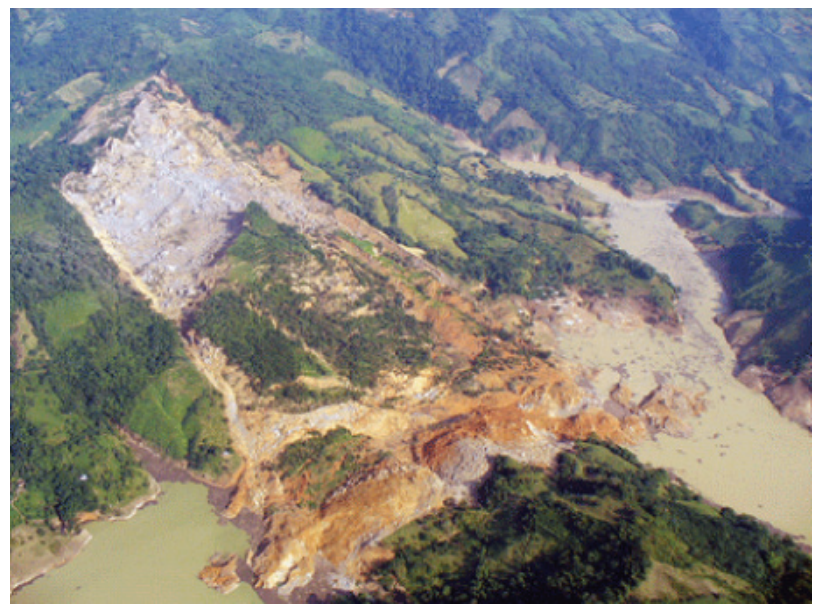

Fuente: Comisión Federal de Electricidad (CFE)

Figura 16. Fotografía aérea del deslizamiento de talud

Aquellos taludes susceptibles al deslizamiento y que ponen en peligro vidas humanas es conveniente protegerlos contra el humedecimiento excesivo. Esto se puede realizar mediante la colocación de drenes y/o membranas que canalicen el agua de lluvia hacia otras zonas.

En este trabajo se establece una metodología que puede emplearse para determinar el riesgo de deslizamiento de un talud ante la ocurrencia de lluvias extraordinarias.

\section{Agradecimientos}

Los autores agradecen al Ing. Eduardo Mora Serafín y a la Universidad Juárez Autónoma de Tabasco, que a través de su División Académica de Ingeniería y Arquitectura, representada por el M. en C. Emmanuel Mungía Balvanera prestó un valioso apoyo para la toma de muestras inalteradas. Al Ing. Javier Espinosa del departamento de climatología de la CONAGUA, al Ing. Gerardo Cruz Velásquez, Residente General de la Contingencia río Grijalva, a cargo de la Comisión Federal de Electricidad (CFE), por su importante apoyo y las facilidades brindadas para el desarrollo de este trabajo.

\section{Referencias}

Alonso E., Gens A., Lloret A., Delahaye C. Effect of Rain Infiltration on Stability of Slopes, 2, pp. 241- 249, en: Unsaturated Soils, Alonso \& Delage (eds), 1995.

Bujang B.K. American Journal of Environmental Sciences, University of Putra, Malaysia, 2006.

Cancelli A. Evolution of Slopes in Over-Consolidated Clays, en: Proc 10th. International Conference on Soil Mechanics and Foundation Engineering, Stockholm, 15-19 June ,1981, 3, pp. 377-380. Publ. Rotterdam, 4. A. Balkema.

Casagli N., Dapporto S., Ibsen M.L., Tofani V., Vannocci P. Analysis of the Landslides Triggering Mechanism During the Storm of 20th-21st November 2000, en: Northern Tuscany, Landslides, 3, pp. 13-21, 2005.

Cervantes-Albarrán J.R. Modelación del flujo de agua en suelos expansivos. Tesis (Maestría en ciencias). Terminal mecánica de suelos. Universidad Autónoma de Querétaro. 2001.

Ching-Chuan H., Chien-Li L., Jia-Shiun J., Lih-Kang H. Internal Soil Moisture Response to Rainfall-Induced Slope Failures and Debris Discharge. Engineering Geology, 101:134-145. 2008.

Cho S.E., Lee S.R. Instability of Unsaturated Soil Slopes Due to Infiltration. Computers and Geotechnics, 28:185-208. 2001.

Cho S.E., Lee S.R. Evaluation of Surficial Stability for Homogeneous Slopes Considering Rainfall Characteristics. Journal of Geotechnical and Geoenvironmental Engineering, 128(9):756-763. 2002.

Comisión Federal de Electricidad (CFE). Residencia General Contingencia Río Grijalva, en: Ing. Gerardo Cruz Velásquez (ed). San Juan de Grijalva, Ostuacán, Chiapas, 2008.

Collins B.D., Znidarcic D. Stability Analyses of Rainfall Induced Landslides. Journal of Geotechnical and Geoenvironmental Engineering, 130(4):362-372. 2004.

Danjon F., Barker D.H., Dreshage M., Stokes A. Using Three-Dimensional Plant Root Architecture in Models of Shallow-Slope Stability. Annals of Botany, Oxford Journals, 101(8):1281-1293. 2008.

Evaluación para Presidencia de la Republica. $2^{\underline{a}}$ reunión de evaluación con el c. Presidente de la República, Lic. Felipe Calderón Hinojosa, 2007. 
Flores-Berrones R., Alva-García F., Li-Liu X. Efecto del flujo de agua en la estabilidad de taludes. Ingeniería hidráulica en México, 18(2):35-52. 2003.

Fredlund D.G., Rahardjo H. Soils Mechanics for Unsaturated soils, 1985, $224 \mathrm{p}$.

Gardner W.R. Some Steady-State Solutions of the Unsaturated Moisture Flow Eequation with Application to Evaporation from a Water Table. Soil science, 85:228:232. 1958.

Geo-Slope. SLOPE/W. Manual de usuario para análisis de estabilidad de talud, versión 3, Calgary, Alberta, Canada. Geo-Slope International Ltd., 1995.

Jaehong K., Sangseom J., Seongwan P., Jitendra S. Influence of Rainfall-Induced Wetting on the Stability of Slopes in Weathered Soils. Engineering Geology, 75:251-262. 2004.

Juárez-Badillo E., Rico-Rodríguez A. Pruebas de compresión triaxial, anexo XII-c, en: Fundamentos de mecánica de suelos, tomo 1, México, D.F., Limusa, 1998, pp. 431-452.
Normaniza O., Faisal H.A., Barakbah S.S. Engineering Properties of Leucaena leucocephala for Prevention of Slopes Failure. Ecological Engineering, 32.215-221. 2008.

Pires L.F., Cássaro F.A.M., Reichardt K., Bacchi O.O.S. Soil Porous System Chamges Quantified by Analyzing Soil Water Retention Curve Modification. Soil \& Tillage Research, 100:72-77. 2008.

PlaxFlow. Plaxflow Scientific Manual, versión 1, Países Bajos, R.B.J. Brinkgreve Delft University of Technology \& PLAXIS b.v. 2003.

PlaxFlow. Manual de información general, versión 1, Países Bajos R.B.J. Brinkgreve Delft University of Technology \& PLAXIS b.v. 2003.

Tohari A., Nishigaki M., Komatsu M. Laboratory Rainfall Induced Slope Failure with Moisture Content Measurement. Journal of Geotechnical and Geoenvironmental Engineering. 2007.

Xie M., Esaki T., Cai M. A Time-Space Based Approach for Mapping Rainfall-Induced Shallow Landslide Hazard. Environmental Geology, 46:840-850. 2004.

\section{Semblanza de los autores}

René Sebastián Mora-Ortiz. Obtuvo el grado de ingeniero civil por la Universidad Juárez Autónoma de Tabasco en el 2006, recibió el reconocimiento como mejor promedio de la generación 2001-2006. Actualmente estudia el posgrado en mecánica de suelos en la Universidad Autónoma de Querétaro. Participó en el 4º Congreso Internacional de Ingeniería organizado en la ciudad de Santiago de Querétaro, Querétaro, con el artículo: "Análisis de un terraplén carretero desplantado sobre suelo pantanoso", el cual fue publicado en las memorias. Participó como co-autor del artículo científico "Resistencia al esfuerzo cortante de los suelos no saturados", el cual fue publicado en las memorias de la XXIV Reunión Nacional de Mecánica de Suelos, que se celebró en la ciudad de Aguascalientes, Aguascalientes.

Eduardo Rojas-González. En 1980 realiza la especialidad en hidráulica en Toulouse, Francia, posteriormente se trasladó al Instituto de Mecánica de Grenoble, en donde realizó los estudios de maestría (1982) y doctorado (1984) en mecánica de suelos. Fue profesor asociado del Instituto Técnico Universitario en 1983 y 1984, año en el que se integró como investigador del Instituto de Ingeniería de la UNAM en donde permanece hasta 1996. Fue profesor de la División de Estudios de Posgrado de la UNAM y asesor de investigación de la Fundación Barros Sierra en 1987 y 1988. Fue jefe del área de mecánica de suelos de la División de Estudios de Posgrado de la UNAM de 1993 hasta 1996. Ha recibido múltiples distinciones como: Investigador Nacional nivel 1 por el Sistema Nacional de Investigadores, el Premio "González Flores" en investigación por la Sociedad Mexicana de Mecánica de Suelos en 1992, la mejor predicción para el comportamiento de pilotes en un campo de prueba, Simposio sobre Predicción y Teoría, Universidad de Oxford, Inglaterra. Asimismo el $2^{\circ}$ lugar del Premio Alejandrina en Investigación (Ciencia y Tecnología), UAQ, 2000, el $1^{\underline{0}}$ y $2^{\mathrm{o}}$ lugar de la misma distinción en 2002 y 2004, respectivamente. 